\title{
Adaptación y estudio preliminar de un test breve para evaluar la eficacia lectora (TECLE)
}

\author{
Adaptation et étude préliminaire d'un test court évaluant la lecture (TECLE) \\ Adaptação e estudo preliminar de um teste breve para avaliar a eficácia leitora (TAEL) \\ Adaptation and preliminary study of a brief test to evaluate reading efficacy (TECLE)
}

\author{
Aldo Ferreres ${ }^{1,2}$, Valeria Abusamra ${ }^{1,2}$, Andrea Casajús ${ }^{1,2}, \&$ Nancy China ${ }^{1,2}$ \\ ${ }^{1}$ Universidad de Buenos Aires, Argentina. ${ }^{2}$ Hospital Eva Perón, Argentina.
}

Agradecimientos: Este trabajo ha sido parcialmente financiado por el subsidio UBACYT P008.

\section{Resumen}

\begin{abstract}
En medios hispanohablantes hay muy pocas pruebas breves y de aplicación colectiva destinadas a evaluar la eficacia lectora y a detectar de manera económica dificultades en la misma. Contar con este tipo de técnicas resulta importante para fines educativos, clínicos y de investigación. Uno de los test que responde a esos requerimientos es el TECLE "Test colectivo de eficacia lectora". Los objetivos del presente trabajo fueron adaptar el TECLE a las particularidades léxico sintácticas de Buenos Aires y sus alrededores y realizar un estudio preliminar en población escolar local (693 alumnos de $5^{\circ}, 6^{\circ}$ y $7^{\circ}$ grado) para evaluar el funcionamiento de los ítems y estimar la influencia de las variables sexo, grado, oportunidades educativas y tipo de gestión (estatal o privada). Los resultados mostraron que los ítems tienen un nivel de dificultad adecuado y que el rendimiento en el test está influido por el grado y el nivel de oportunidades educativas pero no por el sexo. Asimismo se observó que cuando se controla adecuadamente el nivel de oportunidades educativas el rendimiento de los alumnos que asisten a escuelas de gestión estatal es superior al de los que asisten a escuelas de gestión privada. Estos resultados preliminares sugieren que la adaptación del test funciona adecuadamente y proveen información relevante para futuros estudios de validez, confiabilidad y normatización.

Palabras-clave: Evaluación; test breve; eficacia lectora; adaptación.
\end{abstract}

\section{Résumé}

En espagnol, il existe très peu de tests courts évaluant la lecture, permettant de détecter les difficultés dans ce domaine, et qui peuvent être administrés collectivement. La disponibilité de ce type d'outil est importante dans les domaines de l'éducation, la clinique et la recherche. L'un des tests disposant de ces qualités est le « Test colectivo de eficacia lectora » (TECLE), créé par Marín et Carrillo (1999). Les objectifs du présent travail étaient doubles : (a) adapter le TECLE aux caractéristiques syntaxiques lexicales de Buenos Aires, et (b) entreprendre une étude préliminaire sur la population locale d'âge scolaire (693 enfants des grades 5,6 et 7) dans le but d'évaluer la validité des et de déterminer l'influence de variables comme le sexe, le grade, les opportunités éducationnelles et le type d'école (publique ou privée). Les résultats ont montré que les items ont un niveau de difficulté approprié et que la performance au test est influencée par le grade et les opportunités éducationnelles, mais pas par le sexe. Il a également été observé que lorsque le niveau des opportunités d'éducation est contrôlé, les enfants qui ont une scolarité dans un établissement public ont de meilleures performances que ceux qui font partie d'un établissement privé. Ces résultats préliminaires suggèrent que l'adaptation du test fonctionne correctement et qu'elle fournit des informations pertinentes pour des études futures de validité, fidélité et standardisation. Mots clés : Évaluation; dépistage ; lecture, adaptation.

Artículo recibido: 13/08/2010; Artículo revisado (primera revisión): 13/09/2010; Artículo aceptado: 08/04/2011.

Aldo Ferreres, Facultad de Psicología, Universidad de Buenos Aires; Hospital Eva Perón. Valeria Abusamra, Facultad de Filosofía y Letras, Universidad de Buenos Aires; Hospital Eva Perón. Andrea Casajús, Facultad de Filosofía y Letras, Universidad de Buenos Aires; Hospital Eva Perón. Nancy China, Facultad de Psicología, Universidad de Buenos Aires; Hospital Eva Perón.

Todo correo relacionado con este artículo debe ser enviado a: Aldo Ferreres, Hospital Eva Peron, Balbín 900, San Martín, Provincia de Buenos Aires (CP 1650), Argentina.

E-mail: aferrere@psi.uba.ar

DOI: $10.5579 / \mathrm{rnl} .2011 .0040$ 
Em espanhol, existem poucos testes breves de avaliação da eficácia leitora e de detecção das dificuldades nessas áreas que podem ser aplicados coletivamente. A disponibilidade desse tipo de protocolo é importante para fins educacionais, clínicos e de pesquisa. Um dos testes que possui esses requisitos é o 'Teste de avaliação da eficácia leitora' (TAEL). Os objetivos do presente trabalho foram adaptar o TAEL às particularidades lexicais sintáticos de Buenos Aires e cidades vizinhas e realizar um estudo preliminar em uma população escolar (693 alunos de $5^{\mathrm{a}}, 6^{\mathrm{a}}$ e $7^{\mathrm{a}}$ série) para avaliar o funcionamento dos itens e estimar a influência das variáveis sexo, série, oportunidades educativas e tipo de escola (pública ou privada). Os resultados demonstraram que os itens têm um nível de dificuldade adequado e que o rendimento no teste é influenciado pela série e nível de oportunidade educativa, mas não pelo sexo. Assim mesmo, foi observado que quando se controla adequadamente o nível de oportunidades educativas, o rendimento dos alunos que freqüentam escolas públicas é superior aos que freqüentam escolas privadas. Esses resultados preliminares sugerem que a adaptação do teste funciona adequadamente e fornece informações relevantes para futuros estudos de validade, confiabilidade e normatização.

Palavras-chave: Avaliação; teste breve; eficácia leitora; adaptação.

\begin{abstract}
In Spanish, there are very few brief tests to evaluate reading efficacy and to detect difficulties in this area that can be applied collectively. The availability of this type of tool is important for educational, clinical, and research purposes. One of the tests that meet these requirements is the 'Test colectivo de eficacia lectora' (TECLE). The objectives of the present work were to adapt the TECLE to the lexical syntactic features of Buenos Aires and to undertake a preliminary study of the local school-age population (693 students in grades 5, 6, and 7) in order to evaluate how the items operate and to consider the influence of variables such as sex, grade, educational opportunities, and type of school (state or private). The results showed that the items have a suitable level of difficulty and that performance at the test is influenced by grade and the level of educational opportunities but not by sex. It was also observed that when the level of educational opportunities is suitably controlled, students that attend public schools perform better than those who attend private schools. These preliminary results suggest that the adaptation of the test works suitably and provides relevant information for future studies of validity, reliability, and standardization.
\end{abstract}

Keywords: Evaluation; screening; reading efficacy; adaptation.

En la evaluación de la lectura en escolares es necesario distinguir entre instrumentos exhaustivos e instrumentos de detección o rastreo (screening). Los instrumentos exhaustivos están destinados a valorar el estado de los procesos lectores de manera amplia y profunda, son de administración individual y se aplican cuando ya existe la sospecha de un trastorno del aprendizaje de la lectura. La extensión de estos instrumentos se justifica en la necesidad de fundamentar el diagnóstico clínico y sobre todo de precisar las causas que subyacen al problema de aprendizaje. En los últimos años han surgido en el medio hispanohablante un número de baterías de esta naturaleza teóricamente inspiradas en los avances introducidos por la neuro y la psicolingüística en la investigación de las alexias y dislexias. Entre otros, se destacan las técnicas PROLEC (Cuetos, Rodríguez \& Ruano, 1996), PROLEC-SE (Ramos \& Cuetos, 1997) y PROLEC-R (Cuetos, Rodríguez, Ruano \& Arribas, 2007) con normas para España y el LEE (Defior et al., 2006) que cuenta con datos normativos para España y Argentina.

Como todo instrumento exhaustivo las mencionadas baterías contienen un número importante de pruebas y estímulos que proveen información detallada de gran utilidad para el diagnóstico y para la intervención en casos clínicos. Sin embargo, estos instrumentos resultan poco apropiados como pruebas de screening debido a que su extensión y aplicación individual los hacen demasiado costosos para evaluar una población amplia de manera rápida. A esto se suma que, en general, cada una de las pruebas de estas baterías está destinada a indagar sobre el estado de un número reducido de procesos lectores más que a valorar la lectura de manera global.

Recientemente se ha comenzado a dar respuesta, en nuestro medio, a la necesidad de desarrollar pruebas de screening breves, que puedan ser aplicadas colectivamente y permitan evaluar la habilidad lectora de manera global y económica. Este tipo de técnicas resulta relevante para fines educativos, clínicos y de investigación. En educación, un instrumento de esta naturaleza facilita la evaluación de los progresos en lectura, los estudios comparativos y la detección de sujetos en riesgo que pudieran requerir más entrenamiento y atención docente. En clínica, podría ser aplicado como paso previo al inicio de una evaluación exhaustiva, es decir, como recurso complementario, no alternativo a las baterías exhaustivas existentes. En investigación, contar con una primera medida de la habilidad lectora es un requerimiento indispensable para organizar las muestras de estudio.

Una prueba que responde a los mencionados requerimientos es el "Test colectivo de eficacia lectora, TECLE" desarrollado por Marín y Carrillo (1999) siguiendo la estructura del test en francés D-OR-LEC de Lobrot (1980). Así como las baterías exhaustivas están destinadas a obtener un detalle analítico del estado de los procesos de la lectura, el TECLE busca incluir en una única medida, a la que denomina eficacia lectora, los principales parámetros que la controlan: la precisión y velocidad en la decodificación, la comprensión del significado de la oración y la administración de los recursos cognitivos sin intentar un análisis diferenciado de estos procesos. La prueba consiste en completar oraciones en las que falta la última palabra mediante selección (múltiple choice) entre cuatro opciones que incluyen la palabra correcta, otra palabra similar pero incorrecta, una no-palabra (o pseudopalabra) fonológicamente próxima y una no-palabra ortográficamente próxima, todo dentro de un tiempo límite estipulado. Las habilidades que teóricamente contribuyen al rendimiento en esta tarea son al menos tres: en primer lugar, la velocidad y precisión en el reconocimiento léxico y la decodificación que influyen tanto en la lectura de la oración como en el análisis rápido de las opciones; en segundo lugar, el procesamiento sintáctico y semántico de la oración que restringe el número de respuestas correctas posibles, y en tercer lugar, la administración de los recursos cognitivos que permite adecuarse a los requerimientos de la tarea para avanzar en su resolución. El formato del test y la posibilidad de tomarlo colectivamente, lo hacen adecuado para una prueba de screening. 
Se han realizado varios trabajos con el TECLE. En Uruguay se lo utilizó para caracterizar buenos y malos lectores en alumnos de escuela primaria (Cuadro, 2005), para seleccionar las muestras en la investigación de subtipos de lectores retrasados (Cuadro \& Marín, 2007) y recientemente se han publicado las normas a partir de una amplia muestra de alumnos que asistían a escuelas privadas de la ciudad de Montevideo (Cuadro, Costa, Trías \& Ponce de León, 2009). La prueba ha sido utilizada también en Chile (Bravo, Villalón \& Orellana, 2003) y en España (Calvo, 1999; Carrillo \& Alegría, 2009). En Argentina se estudió la correlación del TECLE con la comprensión de textos evaluada con el test Leer para Comprender TLC (Abusamra, Ferreres, Raiter, De Beni \& Cornoldi, 2010) en alumnos de primaria y se observó una correlación positiva y significativa de fuerza moderada (Ferreres et al., 2010b); asimismo se aplicó el TECLE en sujetos adultos con y sin lesión cerebral para comparar el rendimiento en distintas tareas de lectura y se observó que también en adultos correlacionaba con las tareas de comprensión de textos y resultaba una medida sensible para detectar las dificultades en lectura provocadas por lesión cerebral (Ferreres, Sampedro, Abusamra, Casajús \& Cartoceti, 2010c).

Los objetivos del presente trabajo fueron dos: 1) adaptar el TECLE de Marín y Carrillo (1999) a las particularidades léxico-sintácticas de Buenos Aires y sus alrededores y, 2) realizar un estudio con población escolar para evaluar el funcionamiento de los ítems y estimar la influencia de las variables sexo, grado, oportunidades educativas y tipo de gestión (estatal/privada) que permita, $a$ posteriori, efectuar el diseño definitivo del test y el estudio normativo sobre la base de datos empíricos.

\section{Método}

El TECLE consiste en la presentación por escrito de 64 oraciones en las que falta la palabra final, seguidas por cuatro opciones de respuesta. Ejemplo:

\section{Marca con una $X$ la palabra que completa la oración:}

\section{El caballo tenía la pata...}

$\square$ ropa $\square$ rota $\square$ rofa $\square$ rona

La tarea del niño consiste en marcar, en el menor tiempo posible, la alternativa que completa correctamente la oración (rota, en el ejemplo) descartando tres distractores que siguen los siguientes criterios: un distractor léxico (ropa) cuya inserción al final de la oración resulta inadecuada desde el punto de vista semántico o sintáctico, un distractor fonológico (rona) que es una no-palabra construida mediante el procedimiento de sustituir una letra por otra cuyo sonido es similar a alguno de los fonemas de la palabra blanco, un distractor ortográfico (rofa) cuya cercanía con el blanco está dada por la similitud desde un punto de vista visual (se sustituye una letra por otra con características ortográficas similares (/b/ por /d/ o /l/ por /t/, etc.). El tiempo de administración está limitado a cinco minutos y las respuestas posibles son: acierto (elige el blanco), error (elige un distractor) u omisión (no responde el ítem). La puntuación individual resulta de la suma de respuestas correctas que cada participante alcanza en los cinco minutos. Esta prueba se puede administrar en forma individual o colectiva.

\section{Adaptación}

Para lograr una versión adaptada a las particularidades léxico-sintácticas de Buenos Aires y sus alrededores se tomó como base la versión uruguaya del TECLE (Cuadro, 2005) que es casi idéntica a la versión peninsular. El análisis de los estímulos originales del español peninsular mostró que varios de ellos no se ajustaban al español de Buenos Aires. En algunos se utilizaban estructuras sintácticas (por ejemplo la oración "Que tu barco zarpe mañana es imposible") y/o palabras ("rotulador", "alcachofa") que son infrecuentes en el español local. En tales casos se decidió utilizar las formas gramaticales y léxicas más habituales en nuestro medio ("Es imposible que tu barco zarpe mañana", "marcador" y "alcaucil" respectivamente). También se cambiaron los ítems en $2^{\circ}$ persona porque nuestro dialecto utiliza la persona "vos" en lugar de "tú" y "ustedes" en lugar de "vosotros". Por último, se prefirió el tiempo verbal pretérito usado en la variedad rioplatense ("Con el lápiz que me trajiste, no pude escribir") en lugar del pretérito perfecto típico del español peninsular ("Con el lápiz que me has traído no he podido escribir"). En total se modificaron 52 de los 64 ítems de los cuales 40 fueron cambios menores y consistieron en: 1) modificación de la oración manteniendo las opciones de respuesta (17 ítems), 2) modificación de las opciones de respuesta manteniendo la oración original (4 ítems), 3) modificación tanto de la oración como de las alternativas de respuesta (19 ítems). Sólo 12 de los cambios fueron mayores y consistieron en el reemplazo de ítem completo (ver ejemplos de los cambios en el anexo I).

Muestra

Para seleccionar una muestra lo más representativa posible de la población escolar de la ciudad de Buenos Aires y alrededores se realizó un muestreo no aleatorio estratificado por cuotas según zona de residencia y tipo de gestión educativa (privada o estatal) siguiendo como referencia los datos censales del año 2001 proporcionados por el Instituto Nacional de Estadísticas y Censos (INDEC). La muestra incluyó alumnos de $5^{\circ}, 6^{\circ}$ y $7^{\circ}$ de escuelas primarias de la Ciudad de Buenos Aires que al momento de la toma (año 2008) eran equivalentes, en la progresión de los cursos y en la edad de los alumnos, a $5^{\circ}$ y $6^{\circ}$ grado de primaria y a $1^{\circ}$ año de ESB (Escuela Secundaria Básica) de la Provincia de Buenos Aires. La prueba se aplicó a todos los alumnos presentes el día de la evaluación en los grados y escuelas seleccionados. Posteriormente se descartaron las pruebas de los alumnos que quedaran encuadrados en alguno de los siguientes criterios de exclusión: repetición de grado, antecedentes de enfermedad neurológica o psiquiátrica, trastornos del aprendizaje, trastornos sensoriales o lengua materna diferente del español. Esta muestra inicial quedó conformada por 693 alumnos. Una vez efectuada la puntuación, se aplicó un segundo procedimiento de exclusión que consistió en calcular la media y el desvío estándar de la puntuación por grado y a continuación, se descartaron aquellos casos cuya puntuación individual fuera menor al valor de la media menos dos desvíos estándar del grado correspondiente. Esto llevó a eliminar otros 12 casos con lo que la muestra final quedó 
conformada por 681 alumnos. Las tablas 1 y 2 contienen la distribución de los alumnos según zona por sexo y por tipo de gestión (estatal o privada) de la muestra final.

Tabla 1

Distribución de los alumnos de la muestra según la zona y el sexo

\begin{tabular}{lcccccc}
\hline \multicolumn{1}{c}{ Sexo } & \multicolumn{2}{c}{ Mujeres } & \multicolumn{2}{c}{ Varones } & \multicolumn{2}{c}{ Totales } \\
\hline \multicolumn{1}{c}{ Zona } & $n$ & $\%$ & $n$ & $\%$ & $n$ & $\%$ \\
\hline CABA & 45 & 56,3 & 35 & 43,8 & 80 & 100,0 \\
GBA Norte & 103 & 48,6 & 109 & 51,4 & 212 & 100,0 \\
GBA Oeste & 71 & 45,2 & 86 & 54,8 & 157 & 100,0 \\
GBA Sur & 116 & 50,0 & 116 & 50,0 & 232 & 100,0 \\
Total & 335 & 49,2 & 346 & 50,8 & 681 & 100,0 \\
\hline
\end{tabular}

Nota. CABA=Ciudad Autónoma de Buenos Aires; $\mathrm{GBA}=$ gran Buenos Aires.

Tabla 2

Distribución de los alumnos de la muestra según la zona y el tipo de gestión educativa

\begin{tabular}{lcccccc}
\hline $\begin{array}{c}\text { Tipo de } \\
\text { gestión } \\
\text { educativa }\end{array}$ & Estatal & Privada & \multicolumn{2}{c}{ Total } \\
\hline \multicolumn{1}{c}{ Zona } & $n$ & $\%$ & $n$ & $\%$ & $n$ & $\%$ \\
\hline CABA & 32 & 40 & 48 & 60 & 80 & 100 \\
GBA & 274 & 45,6 & 327 & 54,4 & 601 & 100 \\
Total & 306 & 44,9 & 375 & 55,1 & 681 & 100 \\
\hline
\end{tabular}

Nota. CABA=Ciudad Autónoma de Buenos Aires; $\mathrm{GBA}=\mathrm{Gran}$ Buenos Aires.

\section{Procedimiento}

La prueba se aplicó de manera colectiva, con la maestra presente y en el aula habitual, a todos los alumnos que asistieron el día de la evaluación. En primer lugar, se explicó el procedimiento de toma, advirtiendo que se daría la señal de cuándo comenzar y cuándo finalizar la prueba y se presentaron ejemplos. Inmediatamente después, se distribuyeron las hojas y se procedió a la administración. Los 64 estímulos se presentaron impresos en 4 hojas tamaño oficio de un solo lado.

\section{Análisis de datos}

Se obtuvieron las puntuaciones individuales de los participantes y se calculó el efecto sobre las mismas de las siguientes variables independientes inherentes a los sujetos: sexo, grado, oportunidades educativas y tipo de gestión (estatal o privada).

Se consideró necesario obtener un indicador de las oportunidades educativas debido al efecto que tienen las condiciones socio-económico-culturales y la calidad de la enseñanza sobre los resultados de cualquier proceso de aprendizaje escolar. Sin embargo, no es sencillo operacionalizar algunas de estas variables y además resulta complicado obtener la información relevante relativa a cada alumno. Por ejemplo, un alumno de $5^{\circ}$ grado no puede proporcionar información precisa sobre el nivel económico social o el de educación formal de sus padres, y muchas escuelas por razones de privacidad tampoco pueden proporcionarla. Se decidió entonces categorizar las escuelas participantes en una escala ordinal de tres niveles utilizando datos que, supuestamente, permitirían caracterizar las oportunidades educativas de los niños que asistían a ellas (Ferreres et al., 2010b). Los datos elegidos para construir el indicador fueron: nivel socio-económico predominante de los alumnos asistentes, provisión de merienda reforzada (suplemento alimenticio que se distribuye en escuelas a las que asisten alumnos de bajo nivel socio-económico), porcentaje de repetidores, ausentismo y deserción, equipamiento (biblioteca, laboratorios, sala de computación, gimnasio), jornada simple o doble, dictado de actividades extracurriculares. El dato sobre nivel económico-social que se utilizó es subjetivo (nivel económico-social subjetivamente percibido) y fue proporcionado por un observador externo calificado (el director de la escuela o una autoridad de mayor jerarquía administrativa). Los datos sobre merienda reforzada, equipamiento, actividades extracurriculares, porcentajes de repetidores, ausentismo y deserción fueron proporcionados por los docentes de cada grado y/o la dirección de la escuela. La inclusión del dato sobre doble jornada no implica abrir juicio sobre su eficacia pedagógica, lo que se consideró relevante para el indicador fue que una escuela tuviera o no condiciones (edilicias, presupuestarias, de personal) para implementarla. Los tres niveles quedaron definidos de la siguiente manera: Nivel 1: nivel socio-económico de los alumnos que asisten a la escuela predominante bajo, alta proporción de repetidores por grado (más del 30\%), ausentismo (14\% o más) y deserción, equipamiento deficiente, jornada simple, pocas o ninguna actividad extracurricular, merienda reforzada. Nivel 2: nivel socioeconómico predominante medio, baja proporción de repetidores (entre 6\% y 29\%), bajo nivel de ausentismo (7\% o menos) y sin deserción, equipamiento medio (presente pero no suficiente), jornada simple o doble, actividades extracurriculares. Nivel 3: nivel socio-económico predominante medio o alto, nula o escasa proporción de repetidores (menos del 5\%), sin ausentismo, sin deserción, equipamiento óptimo, doble jornada, actividades extracurriculares. La distribución de los alumnos por grado y nivel de oportunidades educativas se muestra en la Tabla 3.

La significación estadística de los efectos de las variables independientes sexo, grado, oportunidades educativas y tipo de gestión sobre el puntaje obtenido en el TECLE fue evaluada mediante pruebas paramétricas ( $\mathrm{t}$ para dos muestras independientes o ANOVA de una vía) cuando se cumplían los supuestos de normalidad y homocedasticidad y, en caso contrario, se utilizaron las correspondientes pruebas no paramétricas. Para calcular el índice de dificultad de los ítems se dividió el número de aciertos observados en cada ítem por el número total de respuestas registradas para el mismo [aciertos/(aciertos + errores + omisiones)], análisis que se realizó para cada uno de los grados de la muestra.

\section{Resultados}

La media de la puntuación de las mujeres fue ligeramente superior a la de los varones (tabla 4) pero una prueba $\mathrm{t}$ para muestras independientes mostró que esa diferencia no era significativa $(\mathrm{t}(679)=-1,45, \mathrm{p}>0,05)$.

Para verificar si el test era capaz de detectar las modificaciones de la eficacia lectora que se producen con el avance a través de los grados escolares, se calcularon los estadísticos descriptivos de las puntuaciones individuales por 
Tabla 3

Distribución de los alumnos de la muestra según el grado y el nivel de oportunidades educativas

\begin{tabular}{lcccccccc}
\hline Nivel de oportunidades educativas & \multicolumn{2}{c}{ Nivel 1} & \multicolumn{2}{c}{ Nivel 2} & \multicolumn{2}{c}{ Nivel 3 } & \multicolumn{2}{c}{ Total } \\
\hline Grado & $n$ & $\%$ & $n$ & $\%$ & $n$ & $\%$ & $n$ & $\%$ \\
\hline $5^{\circ}$ & 106 & 40,8 & 105 & 40,4 & 49 & 18,8 & 260 & 100 \\
$6^{\circ}$ & 59 & 30,3 & 75 & 38,5 & 61 & 31,3 & 195 & 100 \\
$7^{\circ}$ & 60 & 26,5 & 96 & 42,5 & 70 & 31,0 & 226 & 100 \\
Total & 225 & 33,0 & 276 & 40,5 & 180 & 26,4 & 681 & 100 \\
\hline
\end{tabular}

grado escolar y se testearon con una prueba ANOVA de una vía. Como puede verse en la tabla 5, la media de las puntuaciones aumenta con el grado escolar y la prueba de ANOVA mostró que las diferencias eran significativas (F (2, $678)=81,19, \mathrm{p}<0,001)$. Un análisis post hoc HSD de Tukey verificó que tanto la diferencia entre $5^{\circ}$ y $6^{\circ}$ grado como entre $6^{\circ}$ y $7^{\circ}$ eran significativas $(\mathrm{p}<0,05)$.

Tabla 4

Media y desvío estándar (DE) de la puntuación en el TECLE según el sexo

\begin{tabular}{lccc}
\hline Sexo & Media & $D E$ & $N$ \\
\hline Mujeres & 40,38 & 13,15 & 335 \\
Varones & 38,91 & 13,29 & 346 \\
\hline
\end{tabular}

Nota. $\mathrm{DE}=$ desvío estándar; $\mathrm{n}=$ número de casos.

Tabla 5

Media y desvío estándar $(D E)$ de la puntuación en el TECLE según el grado

\begin{tabular}{lccc}
\hline Grado & Media & $D E$ & $N$ \\
\hline $5^{\circ}$ & 33,72 & 11,55 & 260 \\
$6^{\circ}$ & 38,56 & 12,48 & 195 \\
$7^{\circ} / 1^{\circ}$ EGB & 47,37 & 11,67 & 226 \\
\hline
\end{tabular}

Nota. DE=desvío estándar; $\mathrm{n}=$ número de casos; $\mathrm{EGB}=$ educación general básica.

Tabla 6

Media y desvío estándar $(D E)$ de la puntuación en el TECLE según el nivel de oportunidades educativas

\begin{tabular}{rrrr}
\hline $\begin{array}{c}\text { Nivel de oportunidades } \\
\text { educativas }\end{array}$ & Media & DE & $N$ \\
\hline Nivel 1 & 33,32 & 12,79 & 225 \\
Nivel 2 & 40,83 & 12,17 & 276 \\
Nivel 3 & 45,71 & 11,80 & 180 \\
\hline
\end{tabular}

Nota. DE=desvío estándar; $\mathrm{n}=$ número de casos.

El nivel de oportunidades educativas mostró un efecto significativo sobre el rendimiento en el test TECLE. La tabla 6 muestra que la media de la puntuación es menor en el nivel 1, intermedia en el nivel 2 y mayor en el nivel 3 . Una prueba de ANOVA de una vía mostró que estas diferencias eran significativas $(\mathrm{F}(2,678)=53,12, \mathrm{p}<0,001)$ y el análisis post hoc HSD de Tukey verificó que la diferencia era significativa tanto entre los niveles 1 y 2 como entre los niveles 2 y 3 ( $\mathrm{p}<0,05$ en ambas comparaciones).

Al analizar el rendimiento de los alumnos según el tipo de gestión (estatal o privada) del establecimiento al que asistían, se observó mejor puntuación media en el grupo de gestión privada (media: 42,70; DE: 11,$75 ; \mathrm{N}: 375)$ que en el de gestión estatal (media: 35,88; DE: 13,88; N: 306). La diferencia fue testeada con la prueba no paramétrica $U$ de Mann-Whitney (no se cumplía el supuesto de homocedasticidad) y resultó significativa (Z: -6,71; sig. asintót. bilat. < 0,001). Sin embargo, al analizarse la distribución de los niveles de oportunidades educativas entre escuelas estatales y privadas, se observó que, en nuestra muestra, no había escuelas estatales de nivel 3 ni escuelas privadas de nivel 1 (tabla 7). Este resultado sugirió que el efecto del tipo de gestión podía estar distorsionado por el efecto del nivel de oportunidades educativas. La única manera de comparar el efecto del tipo de gestión sin la distorsión provocada por el de oportunidades educativas fue comparar los alumnos que asistían a escuelas estatales y privadas del nivel 2. En esta comparación, la media de las puntuaciones de los alumnos que asistían a escuelas estatales fue ligeramente mayor que la de los que asistían a escuelas privadas (tabla 8). Nuevamente se utilizó una prueba U de Mann-Whitney (no se cumplía el supuesto de homocedasticidad) y se verificó que la diferencia a favor de la gestión pública, en alumnos de nivel 2 de oportunidades educativas, era significativa (Z: $-2,09$; sig. asintót. bilat. $<0,05)$.

En cuanto a la evaluación de los ítems, los resultados muestran que eran adecuados para la población evaluada. El índice de dificultad en la mayoría de los ítems fue próximo a 1 (menor dificultad) y, naturalmente, esto fue más marcado en $7^{\circ}$ grado. En $5^{\circ}$ grado, 41/64 ítems (64\%) mostraron un índice de dificultad entre los valores 1 y 0,95 es decir que provocaron muy pocos errores u omisiones; en 12 ítems (19\%) el índice osciló entre los valores 0,94 y 0,90 y sólo en 11 ítems (17\%) la dificultad fue un poco mayor ya que el índice varió entre 0,89 y 0,46. En $6^{\circ}$ grado, 49 ítems (77\%) mostraron un índice de dificultad entre 1 y 0,95, 10 ítems $(16 \%)$ entre 0,94 y 0,90 y sólo $5(8 \%)$ entre 0,89 y 0,56 . En $7^{\circ}$ grado, 50 ítems (78\%) estaban en el grupo de baja dificultad, $7(11 \%)$ en el de intermedia y sólo $6(9 \%)$ mostraron un índice entre 0,89 y 0,62 .

\section{Discusión}

El presente estudio realizado con el test TECLE mostró que en alumnos de $5^{\circ}, 6^{\circ}$ y $7^{\circ}$ grado no se observaron diferencias por sexo pero sí por grado y nivel de oportunidades educativas. Asimismo mostró que, dentro del mismo nivel de oportunidades educativas, el rendimiento de los alumnos que asisten a escuelas de gestión estatal es significativamente mejor que el de los alumnos que asisten a escuelas privadas. Finalmente el estudio mostró que la mayoría de los ítems tienen un bajo índice de dificultad.

La ausencia de diferencias por sexo observada en nuestra muestra no pudo ser contrastada con el estudio realizado en Montevideo (Cuadro et al., 2009) debido a que en este último la variable sexo no fue analizada.

La presencia de un efecto significativo del grado (curso escolar) en nuestro estudio es coincidente con el informado en el estudio de Montevideo y sugiere que el rendimiento en el test TECLE es sensible a los cambios operados a través del ciclo escolar. 
Tabla 7

Distribución de los alumnos según el tipo de gestión educativa y el nivel de oportunidades educativas

\begin{tabular}{|c|c|c|c|c|c|c|c|c|}
\hline Nivel de oportunidades educativas & \multicolumn{2}{|c|}{ Nivel 1} & \multicolumn{2}{|c|}{ Nivel 2} & \multicolumn{2}{|c|}{ Nivel 3} & \multicolumn{2}{|c|}{ Total } \\
\hline Tipo de gestión educative & $n$ & $\%$ & $n$ & $\%$ & $n$ & $\%$ & $n$ & $\%$ \\
\hline Estatal & 225 & 73,5 & 81 & 26,5 & -- & -- & 306 & 100,0 \\
\hline Total & 225 & 33,1 & 276 & 40,5 & 180 & 26,4 & 681 & 100,0 \\
\hline
\end{tabular}

Tabla 8

Media y desvío estándar (DE) de la puntuación en el TECLE de los alumnos de nivel 2 según el tipo de gestión educativa

\begin{tabular}{|c|c|c|c|}
\hline Tipo de gestión educative & Media & $D E$ & $N$ \\
\hline Estatal & 42,99 & 14,40 & 81 \\
\hline Privada & 39,93 & 11,02 & 195 \\
\hline
\end{tabular}

Nota. DE=desvío estándar; $\mathrm{n}=$ número de casos.

La presencia de un efecto significativo del nivel de oportunidades educativas no resulta sorprendente porque es conocido que las condiciones socio-económico-culturales y familiares en que se desenvuelve cada niño así como la calidad de la enseñanza formal y no formal que recibe ejercen una fuerte influencia sobre los resultados de cualquier proceso de aprendizaje escolar. Varios estudios realizados en nuestro medio mostraron el efecto de estos factores sobre la alfabetización emergente (Piacente, Marder \& Resches, 2006: Piacente, Talou \& Rodrigo, 1990) así como sobre la alfabetización inicial (Borzone, 1997; Diuk, 2003; Diuk, Signorini \& Borzone, 2000; Piacente, Talou \& Rodrigo, 1990; Querejeta, Piacente, Marder \& Resches, 2005; Signorini \& Piacente, 2003) y sobre la comprensión de textos (Ferreres et al., 2010a). Pero, aunque esperadas, las diferencias encontradas son de una magnitud que sugieren la existencia de un grado tal de segmentación de las condiciones en que se desarrolla la educación que pone en cuestión el papel de la escuela actual en la promoción de la igualdad de oportunidades. Un análisis especializado sobre este aspecto supera los objetivos del presente trabajo y va más allá de la competencia de sus autores pero no deja de plantear un problema apremiante aún para la mirada más superficial. Además, las diferencias por nivel de oportunidades educativas plantean la necesidad de considerar esta variable en el momento de diseñar la muestra para la obtención de las normas.

En nuestra muestra, las escuelas de gestión estatal y privada no mostraron la misma proporción de niveles de oportunidades educativas. Mientras las escuelas estatales pertenecían a los niveles 1 y 2 , las escuelas privadas pertenecían a los niveles 2 y 3. Esta distribución explica que cuando se compararon los rendimientos por tipo de gestión la privada (única que incluía el nivel 3, más alto) mostrara globalmente mejor rendimiento que la estatal (única que incluía el nivel 1, más bajo). Sin embargo, cuando se comparó

el tipo de gestión dentro del nivel 2 de oportunidades educativas, el único en el que estaban presentes ambos tipos de gestión, se observó mejor rendimiento en los alumnos que concurrían a escuelas estatales. Estos resultados muestran que cuando se compara el rendimiento según el tipo de gestión, se puede llegar a conclusiones equívocas si no se consideran simultáneamente las diferencias de oportunidades educativas que existen dentro y entre la gestión estatal y privada.
Finalmente, es necesario efectuar algunas consideraciones sobre el funcionamiento de los ítems sobre la base de los índices de dificultad encontrados. El TECLE es un test con tiempo límite en el que la puntuación depende de la cantidad de respuestas correctas. Si los ítems tienen la misma dificultad, el rendimiento depende sobre todo de la velocidad con que se resuelve cada uno. En los test de velocidad lo ideal es que todos los ítems posean un grado similar de baja dificultad. En nuestra versión del TECLE la mayoría de ellos mostró muy poca dificultad, lo que sugiere que resultaron adecuados para la población estudiada. Sólo unos pocos ítems mostraron índices relativamente altos de dificultad. Estos estímulos podrían modificarse y/o ubicarse al final de la prueba para la versión final y el futuro estudio normativo, de manera de mejorar el funcionamiento del TECLE como prueba de velocidad.

\section{Referências}

Abusamra, V., Ferreres, A., Raiter, A., De Beni, R., \& Cornoldi, C. (2010). Test Leer para Comprender TLC. Buenos Aires: Paidós.

Borzone, A. M. (1997). El proceso de alfabetización en niños pequeños: diferencias socioculturales. Tesis doctoral (inédita). Buenos Aires. UBA.

Bravo, L., Villalón, M., \& Orellana, E. (2003). Predictividad del rendimiento de la lectura: el segundo año básico. Psykhe: Revista de la Escuela de Psicología, 12(2), 29-36.

Calvo, A. (1999). Adquisición de la lectura en lengua castellana: perfiles cognitivos de aprendices con dificultades. Tesis doctoral no publicada.

Carrillo M., \& Alegría J. (2009). Mecanismos de identificación de palabras en niños disléxicos en español: ¿existen subtipos? Ciencias psicológicas, III (2), 135-152.

Cuadro, A. (2005). Caracterización de los malos lectores en relación a los buenos lectores en niños uruguayos. Tesis Doctoral. Universidad de Murcia.

Cuadro, A., Costa, D., Trías, D., \& Ponce de León, P. (2009). Evaluación del nivel lector. Manual técnico del test de eficacia lectora (TECLE) de J. Marín y M. Carrillo. Montevideo: Prensa Médica Latinoamericana.

Cuadro, A., \& Marín, J. (2007). Subtipos de lectores retrasados en español. Ciencias psicológicas, 1-2, 133-148.

Cuetos, F., Rodríguez, B., \& Ruano, E. (1996). PROLEC: evaluación de los procesos lectores. Madrid: TEA.

Cuetos, F., Rodríguez, B., Ruano, E., \& Arribas, D. (2007). PROLEC-R: batería de evaluación de los procesos lectores. Madrid: TEA.

Defior, S., Fonseca, L., Gottheil, B, Adrey, A., Jimenez, A., Pujals, M., Rosa, G., \& Serrano, F. (2006). LEE. Test de lectura y escritura en español. Buenos Aires: Paidós.

Diuk, B. (2003) Procesos de enseñanza y aprendizaje inicial de la lectura y la escritura en niños de sectores urbanos marginales. Tesis Doctoral (inédita). Facultad de Humanidades y Ciencias de la Educación de la U.N.L.P. 
Diuk, B., Signorini, A., \& Borzone, A. M. (2000). Las estrategias tempranas de lectura de palabras en niños de $1^{\circ}$ a $3^{\circ}$ año de educación general básica: un estudio comparativo entre niños procedentes de distintos sectores sociales. Psykhe, 12, 51-62.

Ferreres, A., Abusamra, V., \& Squillace, M. (2010a). Comprensión de textos y oportunidades educativas. Actas del Congreso Iberoamericano de Educación. Buenos Aires 13 al 15 de Septiembre de 2010.

Ferreres, A., Abusamra, V., Squillace, M., Fernández Liporace, M., Cartoceti, R., \& Sampedro, B. (2010b). Análisis psicométricos, datos normativos y otros resultados del test Leer para Comprender. En V. Abusamra, A. Ferreres, A. Raiter, R. De Beni y C. Cornoldi (Eds.) Test Leer para Comprender TLC (131-154). Buenos Aires: Paidós.

Ferreres, A., Sampedro, B., Abusamra, V., Casajús, A., \& Cartoceti, R. (2010c). Alteraciones de la comprensión de textos por lesión cerebral. Memorias II Congreso Internacional de Investigación y Práctica Profesional en Psicología, XVII Jornadas de Investigación, Sexto Encuentro de Investigadores, en Psicología del MERCOSUR, 22, 23 y 24 de noviembre de 2010, TOMO III, 401402.

Lobrot, M. (1980). Lire avec épreuves pour évaluer la capacité de lecture (DOR-LEC). Paris: Editions ESF.

Marín, J., \& Carrillo, M. (1999). Test colectivo de Eficacia Lectora (TECLE). Manuscrito no publicado. Departamento de Psicología Básica y Metodología. Universidad de Murcia.

Piacente, T., Marder, S., \& Resches, M. (2006). Condiciones de la familia y del niño para la alfabetización. La Plata. Comisión de Investigaciones Científicas. Gobierno de la Provincia de Buenos Aires.

Piacente, T., Talou, C., \& Rodrigo, M. (1990). Piden pan... y algo más. Un estudio del crecimiento y desarrollo infantil. Buenos Aires: Siglo XXI-UNICEF.

Querejeta, M., Piacente, T., Marder, S., \& Resches, M. (2005). Características del contexto alfabetizador en familias de diferente nivel socioeconómico. En M. A. Mayor, B. Zubiauz \& E. Diez (Eds.). Estudios sobre la adquisición de las lenguas del Estado (803-818). Salamanca: Ediciones de la Universidad de Salamanca.

Ramos, J. L., \& Cuetos, F. (1997). Batería de Evaluación de los Procesos Lectores en el Alumnado del Tercer Ciclo de Educación Primaria y Educación Secundaria Obligatoria (PROLEC-SE). Madrid: TEA Ediciones.

Signorini, A., \& Piacente, T. (2003). Diferencias individuales en lectura en niños pequeños: vinculando el desarrollo normal y las dificultades. Revista IRICE. Agosto 2003; 49-78. 


\section{Anexo I}

Ejemplos de las modificaciones a la versión original del Tecle

1) Modificación de la oración manteniendo las opciones de respuesta (17 ítems).

Ejemplo:

Estímulo original: Quiso montar en...

$\square$ camello $\quad \square$ camillo $\quad \square$ canello $\quad \square$ canilla

Estímulo nuevo: Quiso subir al...

$$
\square \text { camello } \quad \square \text { camillo } \quad \square \text { canello } \quad \square \text { canilla }
$$

2) Modificación de las opciones de respuesta manteniendo la oración original (4 ítems).

Ejemplo:

Estímulo original: $\quad$ Tu coche es tan viejo que se le caen las...
$\square$ tuercas
$\square$ tuelcas
$\checkmark$ tuestas
tuescas

Estímulo nuevo: Tu coche es tan viejo que se le caen las...

$$
\square \text { puertas } \quad \square \text { pueltas } \quad \square \text { puestas } \quad \square \text { tuerpas }
$$

3) Modificación tanto de la oración como de las alternativas de respuesta (19 ítems).

Ejemplo:

Estímulo original:: $\quad$ Para coser la camisa cogió la aguja, el hilo y el...

$$
\square \text { delal } \quad \square \text { dedos } \quad \square \text { dedal } \quad \square \text { deval }
$$

Estímulo nuevo: Para coserle la camisa buscó el hilo y la...

$$
\square \text { aguja } \quad \square \text { abuja } \quad \square \text { abeja } \quad \square \text { baguja }
$$

4) Cambios que consistieron en el reemplazo de todo el ítem (12 ítems).

Ejemplo:

Estímulo original: Han atrapado un...

$$
\square \text { caslor } \quad \square \text { cantos } \quad \square \text { caspor } \quad \square \text { castor }
$$

Estímulo nuevo: En el hospital le sacaron una...

$$
\square \text { rabiografía } \quad \square \text { raquiografía } \square \text { radiografía } \square \text { radiodifusor }
$$

